\title{
Very affordable post mortem CT angiography kit: Feasibility study using immersion pump and $3 \mathrm{D}$ printed parts
}

\author{
Schweitzer, Wolf ; Enders, Markus ; Thali, Michael
}

\begin{abstract}
The high cost of commercial components and parts for post-mortem computed tomography angiography (PMCTA) has resulted in the development of a very low cost PMCTA-kit. It contains ubiquitous parts from hardware stores, and rapid prototyping derived models for 3D-printing. Design specifications have been tested and improved after finite-element modeling. A case study performed in using the final PMCTA kit parts for a PMCTA shows perfect vascular contrast without relevant leaks.
\end{abstract}

DOI: https://doi.org/10.1016/j.jofri.2018.12.002

Posted at the Zurich Open Repository and Archive, University of Zurich

ZORA URL: https://doi.org/10.5167/uzh-161725

Journal Article

Accepted Version

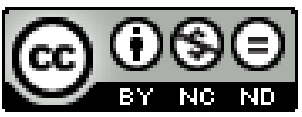

The following work is licensed under a Creative Commons: Attribution-NonCommercial-NoDerivatives 4.0 International (CC BY-NC-ND 4.0) License.

Originally published at:

Schweitzer, Wolf; Enders, Markus; Thali, Michael (2019). Very affordable post mortem CT angiography kit: Feasibility study using immersion pump and 3D printed parts. Journal of Forensic Radiology and Imaging, 16:11-18.

DOI: https://doi.org/10.1016/j.jofri.2018.12.002 


\title{
Very affordable post mortem CT angiography kit: feasibility study using immersion pump and 3D printed parts [PREPRINT]
}

\author{
Wolf Schweitzer*, Markus Enders, Michael Thali \\ Zurich Institute of Forensic Medicine, University of Zurich, \\ Switzerland
}

December 21, 2018

\begin{abstract}
The high cost of commercial components and parts for post-mortem computed tomography angiography (PMCTA) has resulted in the development of a very low cost PMCTA-kit. It contains ubiquitous parts from hardware stores, and rapid prototyping derived models for 3D-printing. Design specifications have been tested and improved after finite-element modeling. A case study performed in using the final PMCTA kit parts for a PMCTA shows perfect vascular contrast without relevant leaks.

Keywords: Post mortem CT-angiography; 3D-design; 3D-printing; forensic imaging; Virtopsy

Citation:

Schweitzer, W., Enders, M., \& Thali, M. (2018). Very affordable post mortem CT angiography kit: feasibility study using immersion pump and 3D printed parts. Journal of Forensic Radiology and Imaging

darticle\{schweitzer2019angio3dprt, title=\{Very affordable post mortem CT angiography

kit: feasibility study using immersion pump and 3D printed parts\}, author=\{Schweitzer,

Wolf and Enders, Markus and Thali, Michael\}, journal=\{Journal of Eorensic Radiology

and Imaging\}, volume $=" 16 "$, pages $=" 11-18 "$, year $=" 2019 "$, issn $=" 2212-4780 "$,

doi = "https://doi.org/10.1016/j.jofri.2018.12.002", publisher=\{Elsevier $\}$
\end{abstract}

\footnotetext{
*Corresponding author: wolf.schweitzer@irm.uzh.ch
} 


\section{Contents}

1 Background 3

2 Methods 5

2.1 Rapid prototyping: 3D design and print . . . . . . . . . 5

2.2 Specific build aspects . . . . . . . . . . . . . . . . 8

2.3 PMCTA method and case scanned for PMCTA . . . . . . . . 9

3 Results 9

3.1 Specification of all parts of the PMCTA kit . . . . . . . . . 9

3.1.1 Ubiquitous hardware store products . . . . . . . . . . . 9

3.1 .2 3D-designed parts . . . . . . . . . . . 11

3.2 PMCTA case study . . . . . . . . . . . . . . . . . 11

4 Discussion $\quad 14$

5 Conclusion $\quad 14$

6 References $\quad 15$

7 Acknowledgements $\quad 18$

8 Conflict of interest $\quad 18$

9 Ethics approval $\quad 18$ 

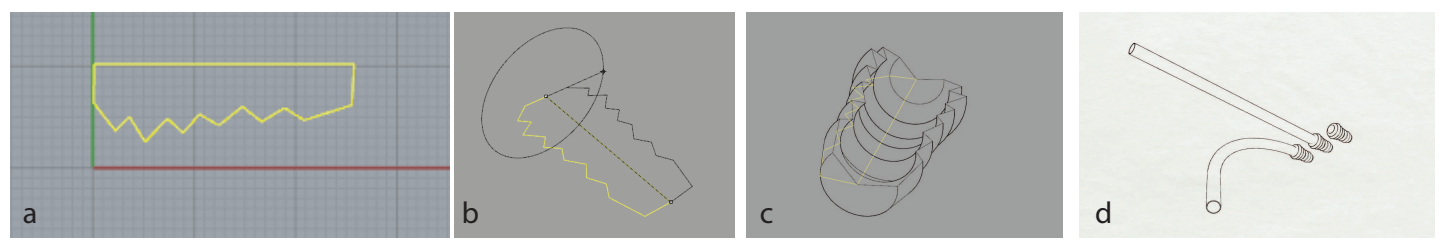

Figure 1: Creating a femoral catheter that attaches to a standard 10/12 silicone tube is greatly facilitated by employing parametric $3 d$ solid modeling starting with a jagged polygon outline $(\boldsymbol{a})$, revolving it $(\boldsymbol{b}, \boldsymbol{c})$ to arrive at a connector piece that can be expanded further with a straight tube that is (virtually) bent (d).

\section{Background}

Postmortem computed tomography angiography (PMCTA) has seen a slow introduction into the field of forensic medicine that so far seemed to be dominated by the installation of heart-lung machines or similar roller pump derived devices into forensic pathology units.

A wider adoption into practice may be desirable seeing as if PMCTA, in general, has become known to help solve particularly tricky forensic pathology cases $[1,2,3$, $4,5,6,7,8,9,10]$ even in decomposed bodies [11].

Strategically, PMCTA as the capacity to place any detectable vessel leak into a time and risk domain that precedes the forensic autopsy and its tissue, organ and vessel dissection and that thus precedes the professional application of sharp force. So proving the existence of vessel injury damage or the absence thereof using PMCTA might have significant legal value.

However, specialized commercial devices can be costly and require dedicated and costly additional installations such as oil separators, servicing, consumables, and maintenance.

A technical revision of that practice showed that a ubiquitous and thus very affordable immersion pump [12], using environmentally acceptable PEG-based iodine contrast admixture [13], achieves superb vascular contrast, also in comparison to a more expensive roller pump $[14,15]$.

Developing PMCTA for successful more full implementation in forensic medicine still appears to require further efforts, to ease forensic pathologists and radiologists into taking it to practice. From organizing and teaching the Virtopsy Courses over the years, we have come to identify some user or consumer beliefs that may directly affect the adoption of PMCTA into forensic pathology practice.

A first belief is that technology, as such, may be hard to understand, generally. The PMCTA process is not at all as difficult as it may appear, as it has been the subject of research since over ten years, even with a recently published atlas (e.g.,t [13, 16].

A second belief is that the various components or parts required to perform an effective PMCTA are difficult, time-consuming and problematic to come by. Generally, 
perceived or expected ease of use might affect the adoption of new technology [17]. A perceived difficulty may be an issue for an examination such as PMCTA that typically is performed occasionally but not routinely. We, therefore, provided an assembly of parts that fit together in the form of a PMCTA kit.

A third belief is that once collected or ordered, bought and ready to use, various parts do not necessarily provide the expected function. That may be a risk for any offlabel experimental use devices; for example, not all pump types are ideal for PMCTA: an immersion pump as the one that we selected for the PMCTA kit, however, provides results that are on par with the results of a roller pump PMCTA [14], even in highpressure low-diameter vascular systems [18]. From there on out, the most critical step towards implementing a routine use of any immersion pump with fitted tubes is the technical specification (material, dimensions/size) of all parts to be used, so they fit and match. With that, perceived efficacy is a relevant factor that impacts technology adoption [17].

A fourth belief is that PMCTA equipment, including consumables, necessarily is very costly. High cost has been identified as an impediment to broader adoption of PMCTA by the Leicester group as a past member of the Technical Working Group Post mortem Angiography Methods (TWGPAM) [19] ${ }^{1}$. Clinical materials such as heart-lung machines but also vascular tourniquet sets and catheters may be medically certified high-end products that are furthermore sold for profit. Our application does not require some of the technical provisions made for clinical use, such as preventing hemolysis (roller pumps) or preventing health issues related to catheter surfaces. With that, the overall cost of the PMCTA kit that we assembled was brought down to around $\sim 120$ USD with two avenues: ubiquitous hardware store products and 3D-printing. With that, we made the full specification of all parts freely available.

A fifth belief is that installation of vascular access may be technically too difficult. However, anyone that can perform an autopsy can also install vascular access for PMCTA.

From the beliefs listed above, it becomes clear that easy to follow instructions, a convincing hand-on-demonstration, and a complete PMCTA kit should be able to address these issues. The kit will be fully specified, financially affordable and fully available concerning both accessible materials and designs.

Centering around the widely available type of immersion pump that had been identified as suitable for PMCTA [12] and that can provide excellent vascular filling [14], such a PMCTA kit was developed. This technical note describes its parts and the first results of a PMCTA using that kit.

\footnotetext{
${ }^{1}$ http://www.postmortem-angio.ch/de/twgpam/twgpam_historical_ members/gb_leicester, retrieved November 20th 2018
} 


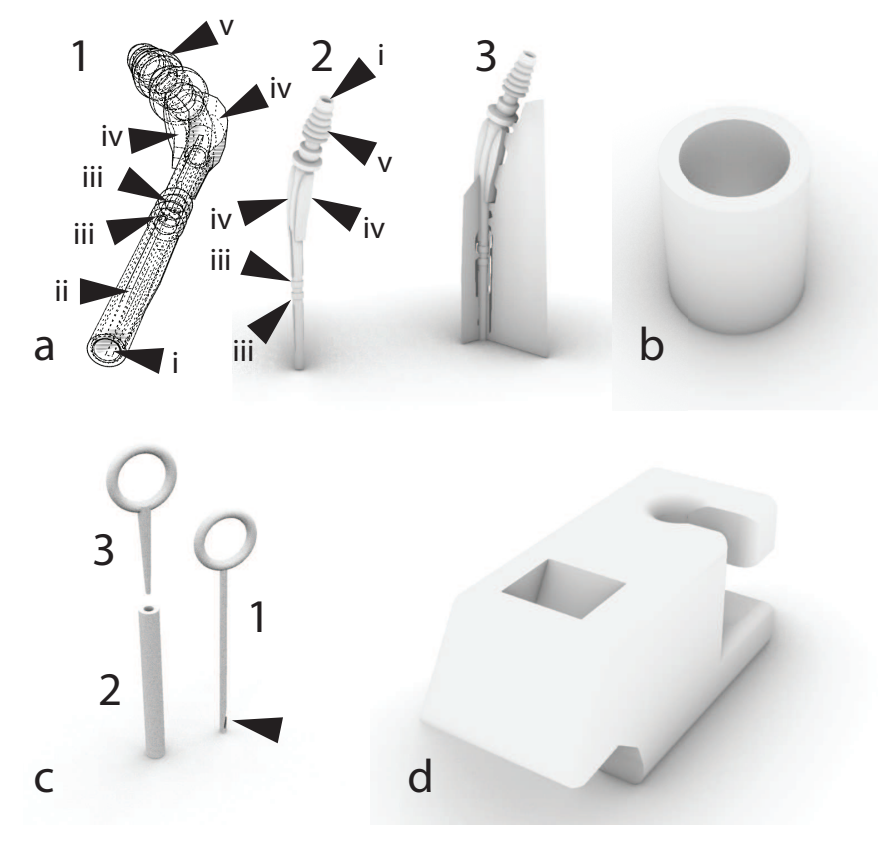

Figure 2: 3d-designed parts for the very affordable PMCTA kit - (a) femoral catheter in 3D-mesh view (1: (i) lumen, (ii) longitudinal reinforcements, (iii) circular tourniquet support (see Fig. 4 and 5 for finite element analysis), (iv) tabs for bending reinforcement, (v) tube connector), in its final form (2) and including (3) 3d-print-relevant customized supports, (b) press-fit cylinder to secure tube to pump and catheter, (c) vascular tourniquet set using a loop or string containing (1) stylet with a small lug (arrowhead) to fix the string before pulling it through the occlusion tube, (2) occlusion tube and (3) a quick release clamp and (d) tube clamp fixture to firmly mount the 10/12 silicone tube to a bucket rim via an ubiquitous spring clamp.

\section{Methods}

\subsection{Rapid prototyping: 3D design and print}

While some parts of the PMCTA kit are ubiquitous products that can be obtained from normal hardware stores, other parts of the kit required a dedicated specific design and build.

The technical design of these parts of this PMCTA kit was performed using software that allows the creation and editing of 3D-models (Blender, Version 2.78, Blender Foundation, Amsterdam, Netherlands; Rhinoceros 3D, Version 5, Robert McNeel \& Associates, Seattle, Washington, USA). On the design level, we employed a hybrid parametric and direct modelling approach. Two particular functions seem noteworthy in the context of building for vascular structures: revolution and bending (Fig. 1). 

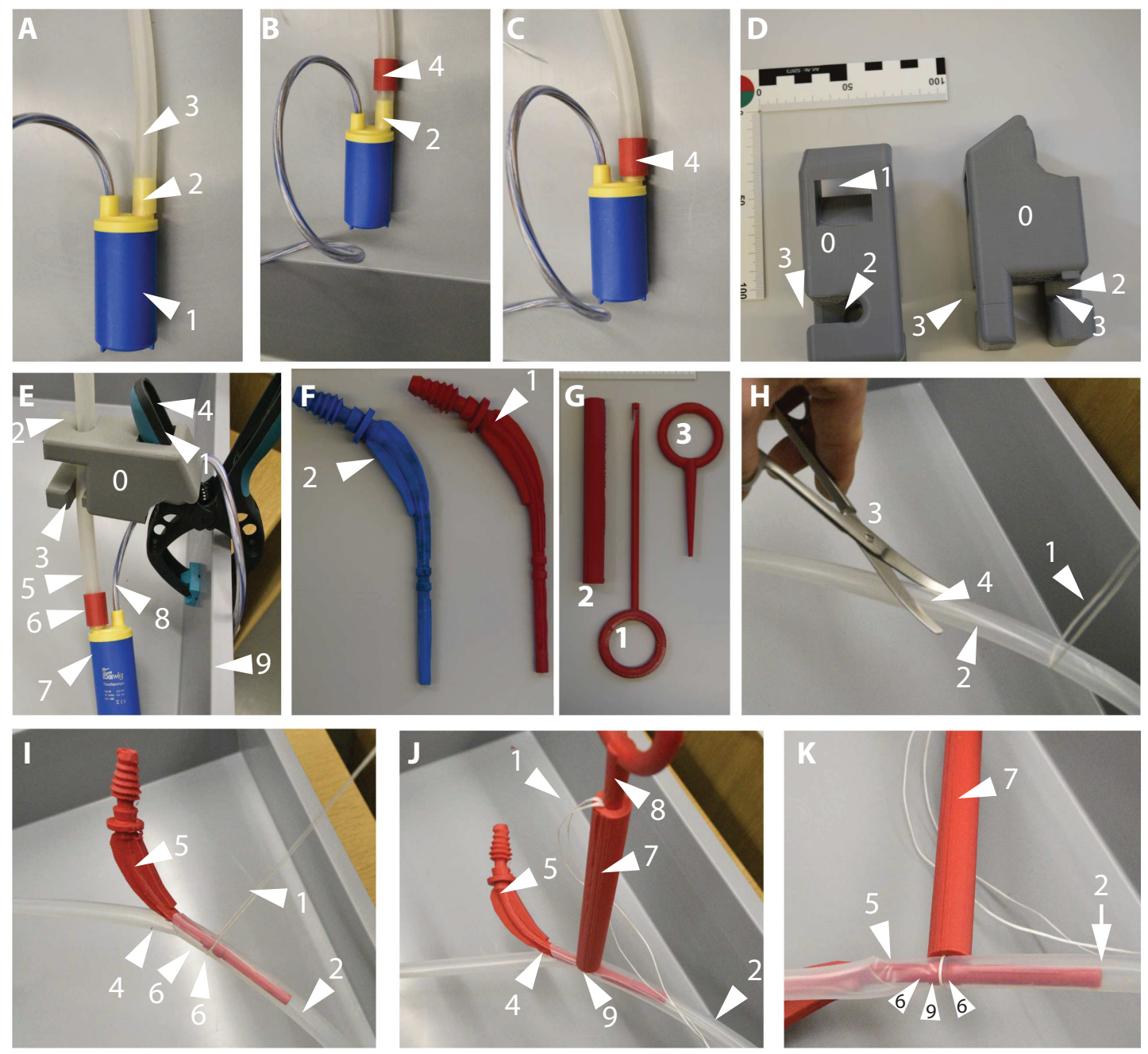

Figure 3: Laboratory setup for testing the handling and for demonstration of practical parts assembly - $(\boldsymbol{A}-\boldsymbol{C})$ Immersion pump (1) with silicone tube (3) pulled over conical extension (2), then sliding press-fit cylinder (4) (also see Fig. 2 b) in place. (D) Tube clamp fixture (0) with opening (1) that slides over spring clamp handle (4) to mount tube (5) without obstructing it, containing longitudinal hole for tube (2) and lateral slide-in gaps (3), effectively allowing immersion pump (7; cable: 8 ) attached with compression fit (6) to be firmly mounted to bucket or container wall (9). (F) Femoral catheters in red (1) and blue (2) color for venous/arterial access distinction after insertion. (G) Vascular tourniquet set parts with (1) stylet, (2) occlusion tube and (3) quick release clamp (see also Fig. 2). (I-K) Insertion of femoral catheter into femoral or iliacal vessel substrate (for better instruction, a thin walled silicone tube is used): after tensioning of vessel substrate (2) with string (1), partial cut (4) with scissors (3) to insert femoral catheter (5), looping string (1) around circular supports (6, but see also Fig. 2/a/iii) of femoral catheter (5) by pulling loop using stylet through occlusion (7) tube, then fixing string using quick release clamp (8), creating firm tension at loop-vessel-catheter junction (9). 


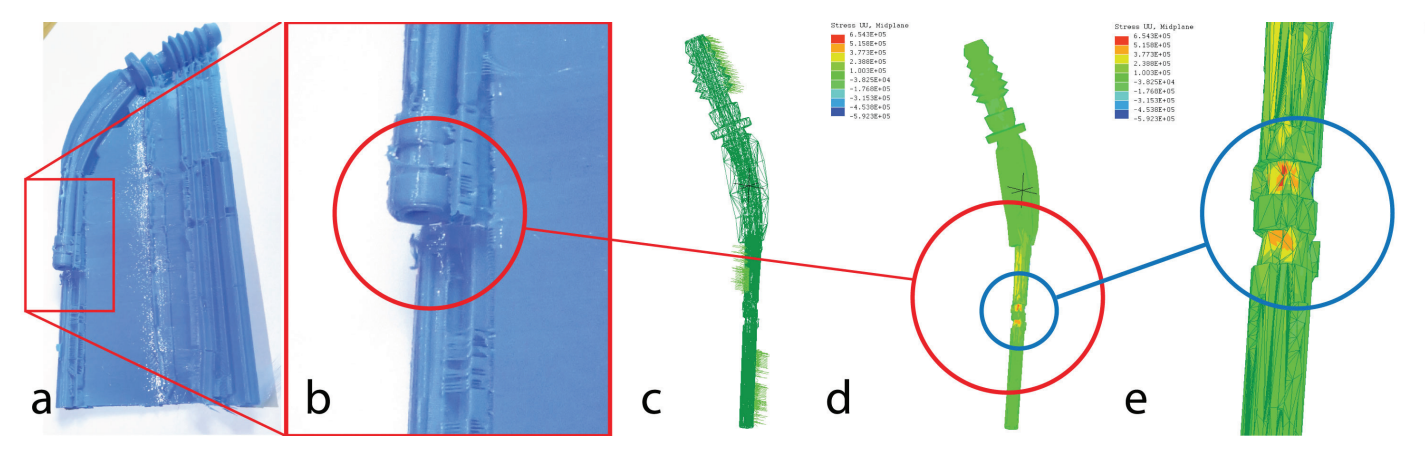

Figure 4: While the current design of the femoral catheter already had seen a number of prior revision cycles to define its current relatively stable shape (given additive manufacturing with layered and thus anisotropic PLA), there is always more left to be desired: any rapid prototyping approach into fast part development is critical also in terms of number and quality of revision cycles. To demonstrate how fault search and analysis can be performed other than by brute force ("just print out and stand on it to see where it breaks"), a more interesting, cheaper and faster (see also Table 1 for print times) approach may evaluate a still somewhat frequently observed break location during manufacturing: $(\boldsymbol{a}, \boldsymbol{b})$ : we frequently $(\sim 8 \%$ of batch produce) observed a break in printed catheters with supports, that broke when attempting manual support structure removal. In finite element analysis for mechanical strain, applying similar virtual forces $(\boldsymbol{c})$ resulted in the identification of a design-specific area of relatively high stress, and then, $(\boldsymbol{d}$, detail in $\boldsymbol{e})$ confirmed a systematic design weakness of particular relevance to the anisotropic nature of the material (only UU stress shown here with relevant values, $V V$ stress (not shown) without relevant results) at precisely that location. A first practical consequence here was to be more careful when manually removing support structures.

These 3D models (Fig. 2, details regarding their specifications see below) then were exported to a widely used, typical standardized file format termed STL (short for stereolithography) where units are generally arbitrary but may be used to encode metric units; we used a millimeter scale. The format is useful also as it allows the models to be shared across different software platforms.

Additive manufacturing derived models such as our PLA builds (polylactic acid, a biodegradable material made from starch or sugar cane) have two inherent issues. Firstly, PLA builds are built layer by layer using support structures to allow the build of overhangs [20]. There is a risk in that supports might be printed where they cannot be removed anymore, and the aspect that removal itself causes significant strain on the remaining plastic of the printed part.

Secondly, printing PLA will result in models that are mechanically anisotropic [21]. From a practical view, this means that their mechanical properties are not the same in all three dimensions. Because the plastic filament is heated and added to the model during the 3D-print process in single layers, the model may have different mechanical properties along its z-axis (i.e., alongside its height relative to the 3D-printer build platform) than in the other directions (which would be in-plane, $x-y$ ). The orientation of the 3D-model relative to the build platform as well as the specific support structure 

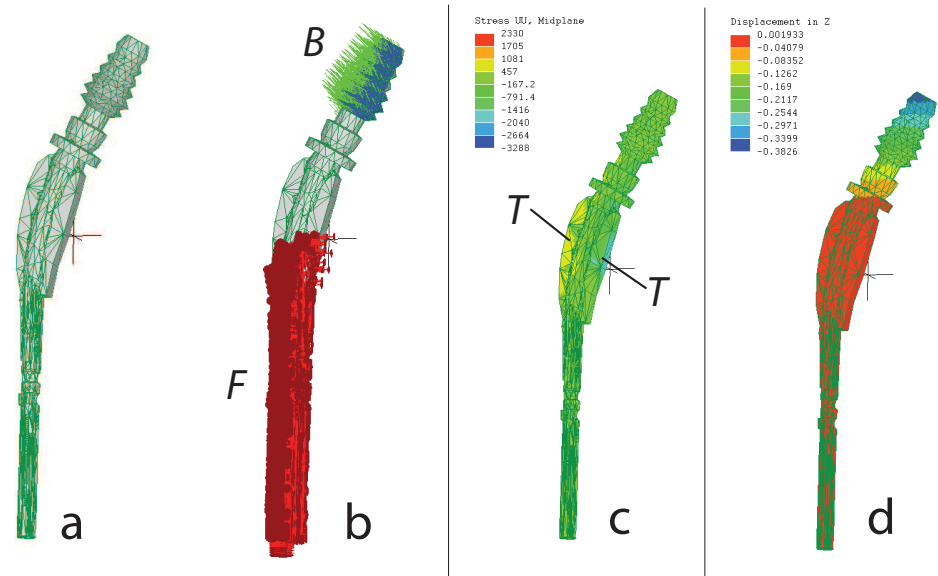

Figure 5: In situ force-specific finite element analysis evaluating mechanical stress when catheter is placed in femoral vessel, using (a) thinned out model mesh structure, with applied forces $(\boldsymbol{b})$ whereas a fixture $(F$, red $)$ and a bending force $(B$, green arrows at tube connector) represent realistic forces acting on an in-situ catheter. The (c) same simulation that adequately modeled a break predilection found during fabrication and 3D post-processing (see Fig. 4) now shows no particular shear stress while displacement under stress forces (d) realistically will allow for some amount of bending. As side remark one should add that from this analysis, it was good to have installed the reinforcing tabs (T): the stress distribution there is notably low.

use thus may or may not be critical depending on particular properties of the shape to be 3D-printed.

The handling of the resulting parts was tested during development first using a clean laboratory dummy setup (Fig. 3).

Then, analysis and testing of rapid prototyping results was performed practically, and also explored using finite element analysis (FEA) (LISA, Sonnenhof Holdings, Mississauga, ON, Canada) (Fig. 4 and 5).

\subsection{Specific build aspects}

3D-printing the femoral catheters involved the print of a circular lumen without added support structures. This required the lumen to be longitudinally oriented in parallel to the $\mathrm{z}$-axis or build direction of the 3D-printer. As that orientation resulted in an otherwise unstable print, specially crafted support structures were added in a lengthy rapid prototyping development cycle here (Fig. 2/3: plate structures).

The rigidity of PLA is not extreme, so the catheters were supplemented with design features to increase their mechanical stiffness within a reasonable extent. Interestingly, we experienced breaks during the clean-up of the 3D-printed models with manual support removal (Fig. 4) while the catheters seemed robust enough for actual use after that (Fig. 6), which had also resulted from simulations using FEA. 


\subsection{PMCTA method and case scanned for PMCTA}

Relevant details of the iodine-based PMCTA procedure and reconstruction software are published in [13, 22, 12, 23].

The body scanned for PMCTA was that of a 30 year-old man who had died of blunt abdominal trauma with kidney rupture and large internal hemorrhage. The body had been without any signs of decomposition. The PMCTA was performed around 18 hours after death.

The technical setup is shown in Fig. 6. The PMCTA region of the femoral catheter insertion is detailed in Fig. 7. The resulting full body PMCTA data is illustrated in Fig. 8.

Plastic instances of the 3D-models that had been designed were realized using PLA (filament diameter: $1.75 \mathrm{~mm}$, manufacturer: Stratasys, Eden Prairie, Minnesota, USA; Young's modulus of 3.11 GPa [24], build thickness of $2 \mathrm{~mm}$ shells) on 3D printers (Replicator, MakerBot, Stratasys, Eden Prairie, Minnesota, USA).

More concisely, a 2X-model (initially built and sold for ABS-printing) was improved for warp free PLA builds covering the whole build plate (Fig. 9) by installing an additional fan (Thingiverse:221306) and a durable hardened glass plate (Creative Tools, Slottsmöllan, Sweden) for production printing. A model of Replicator $+\left(5^{\text {th }}\right.$ generation) was used for development. Printed part characteristics in Table 1.

\section{Results}

\subsection{Specification of all parts of the PMCTA kit}

The total cost of parts was $\sim 120 \mathrm{CHF}$ without and $\sim 170 \mathrm{CHF}$ with a suitcase. The step-wise employment and assembly of the parts for use is explained in Figure 3.

\subsubsection{Ubiquitous hardware store products}

- Suitcase: The use of a suitcase for this kit is not required. After use, the kit parts should be washed. The parts mostly need to be stored so they can dry rather than being enclosed. (Model: Lux Tools AKW 130, ordered from OBI, Winterthur, Switzerland) [hardware store].

- Pump: Technical details see previous publication [12] [hardware store] (Fig. 6/d/5).

- Plugs: Many standard power supplies come with banana plug connectors. So these seem useful to put on the pump cables. (Model specifications: two standard 'banana' plugs, ordered from Conrad Electronic, Switzerland): mounted on the pump cables [hardware store].

- Power supply: The use of a variable rather than fixed voltage power supply has practical advantages, but is not a must (model: PS 1501, EA-Elektroautomatik, Viersen, 

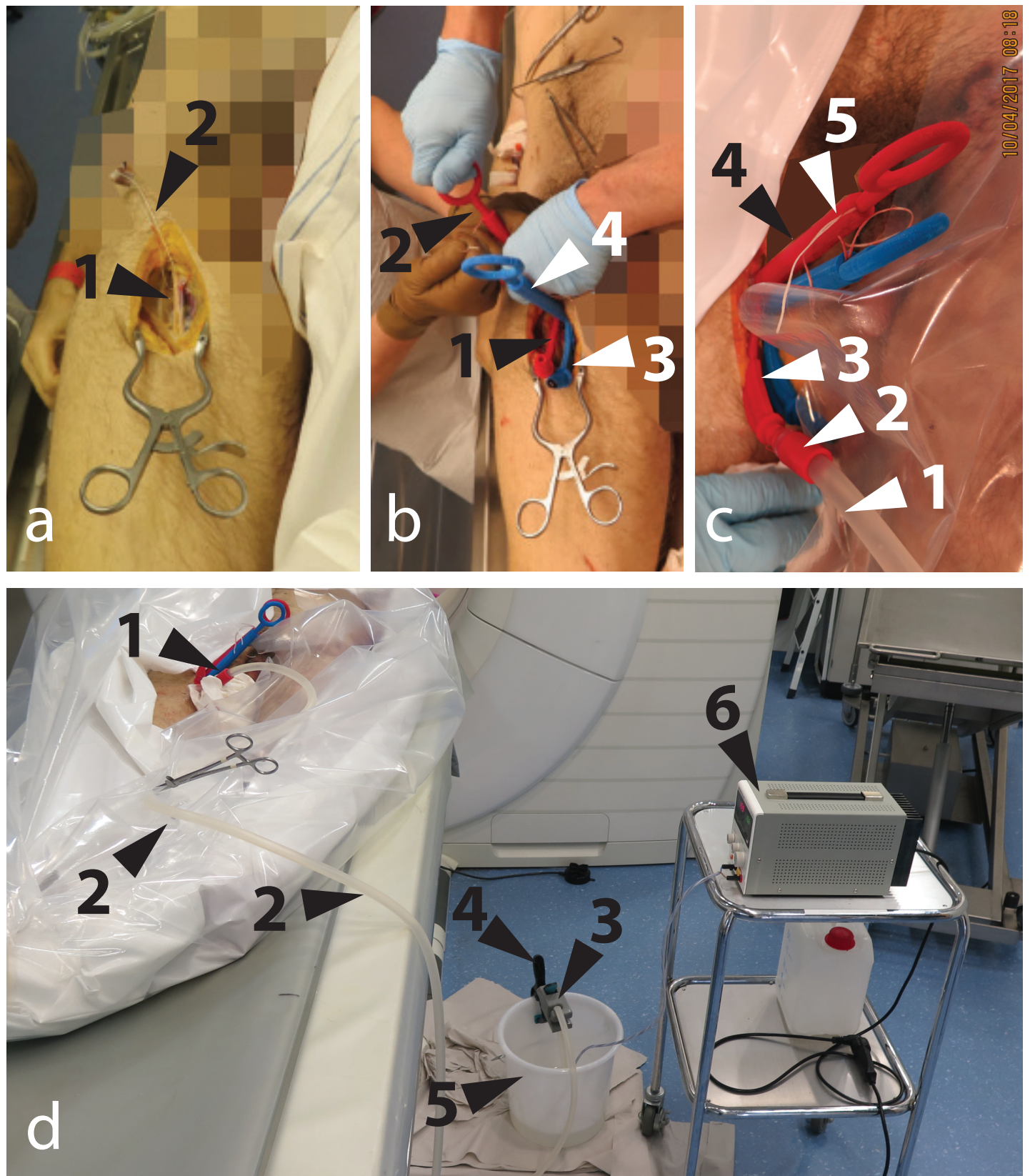

Figure 6: (a): Femoral access after dissection with string loop (2) around femoral artery (1); (b) femoral arterial (1) and venous (3) catheters in place fixed with vascular tourniquet set (2: tourniquet set to fix arterial catheter in place; 4: venous catheter's tourniquet set); (c) tube (1) now attached to catheter, it connects pump with femoral arterial catheter (2 and $\mathbf{3})$ that is fixed to vessel with occlusion tube (4) by way of a string (5); overall view (d) on setup with (1) femoral access [3d printed parts: catheters, vascular tourniquet set], (2) tube, (3) fixture [3d printed] for tube and spring clamp, (4) spring clamp, (5) bucket containing contrast agent admixture and immersion pump at the bottom (hence the term "immersion") and (6) power supply. 
Germany; ordered from Conrad Elektronik, Switzerland): 0-15V with turning knob to vary the voltage [hardware store] (Fig. 6/d/6).

- Tube: (10/12mm silicone tube, ordered from Maag Technic, Switzerland): We put a 10/12 mm silicone tube into the assembly. This tube has an inner diameter of 10 and an outer diameter of $12 \mathrm{~mm}$, that means that its wall is $2 \mathrm{~mm}$ thick. The tube can be attached to the pump where it fits onto the conical extension and to the femoral catheter that is inserted into the femoral vessels (see Fig. 6/c/1 and Fig. 6/d/2). It is held in place more firmly by sliding the enclosed tube push compression fitting (Fig. 2b) over it. To fix the tube to the catheter, both cable binder and push compression fitting cylinder may be used [hardware store]. The tube is held in place at the contrast agent container by a tube fixture (Fig. 2/d, Fig. 3/b, and Fig. 6/d/3).

- Spring clamps (model Wolfcraft 3631, ordered from: Conrad Electronic, Switzerland) (Fig. 6/d/4): Used to fix the tube fixture to a bucket or similar [hardware store].

- String: Sufficient string (i.e., textile string or filament) is used to fix the tourniquet set in place (see Fig. 3) [hardware store].

- Cable binders: These are not absolutely necessary but can be useful as add-on to fix the tube in place (e.g. Fig.6/a/2) [hardware store].

\subsubsection{D-designed parts}

- Femoral catheters(Fig. 2/1, Fig. 6 and 7): Design-wise mechanically the most interesting of the 3D-printed parts, this part combines filigrane design and critical load aspects (Fig. 4 and 5) [3d printed]. See method section for specific build aspects.

- Cylindrical push compression fitting (Fig. 2b): To connect tube to pump and to catheter [3d printed].

- Bucket tube fixture (Fig. 2/d and Fig. 6/d/3): To hold the tube in place while fixing it to a spring clamp that is clamped to the rim of the bucket used for the contrast agent admixture [3d printed]. This part was developed directly from the first paper [12] where it had become clear rather early into first trials that the meager weight of the pump was in the way of it staying at the bottom of the container by itself.

- Vascular tourniquet set (Fig. 2/3, Fig. 3/d and 33/e): the larger-than-for-surgery design of these parts make for easy handling when wearing thick protective gloves.

\subsection{PMCTA case study}

The installation of the femoral access followed the described procedure (Fig. 3) was flawless (Fig. 6). 

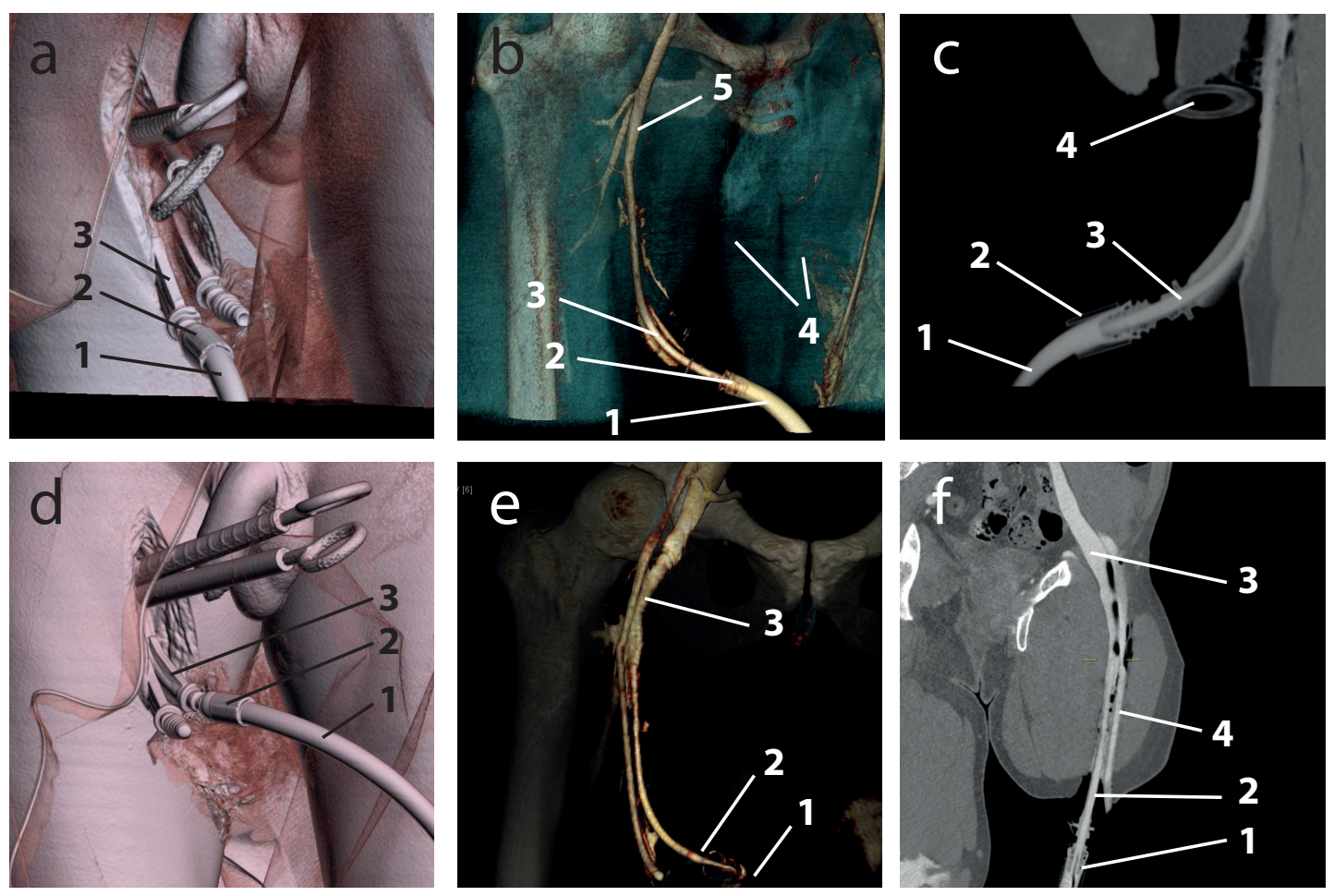

Figure 7: PMCTA-reconstructions of arterial $(\boldsymbol{a}, \boldsymbol{b}, \boldsymbol{c})$ and venous $(\boldsymbol{d}, \boldsymbol{e}, \boldsymbol{f})$ access for documentation and to check for leaks. Arterial access: (a) Skin surface and plastic shown with (1) tube (2) cylindric compression fitting, (3) femoral catheter (VRT). (b, c) Connection of (1) tube with (2) location of compression fitting and (3) catheter and with (4) partly shown vascular tourniquet set's occlusion tube present in the images; (5) contrasted artery (b: VRT; $\boldsymbol{c}: M P R)$. Venous access: (d) Skin surface and plastic shown with (1) tube, (2) compression fitting and (3) catheter. Catheter-vessel interface detailed in $(\boldsymbol{e}, \boldsymbol{f})$ with $(\boldsymbol{1})$ tube, (2) tube-catheter connector, (4) catheter and (3) contrasted femoral vein. -There are minimal amounts of extravascular contrast agent typical for remnants after connecting the tube to the catheter; no larger accumulations present that would indicate faulty material or leakage as all PMCTA scans were obtained after infusion of the contrast agent admixture (and not shortly after start). Also the excellent vascular filling of the PMCTA (Fig. 8) shows an effective contrast agent infusion.

The resulting PMCTA (Fig. 8) showed excellent filling. No particular leaks were noted (Fig. 7).

No mechanical flaws or leaking mainly of the femoral catheters (Fig. 5) were noted during the installation of the femoral access or during the procedure.

The safe manual handling of the immersion pump in conjunction with the PMCTA kit is now easily possible also single-handedly. 

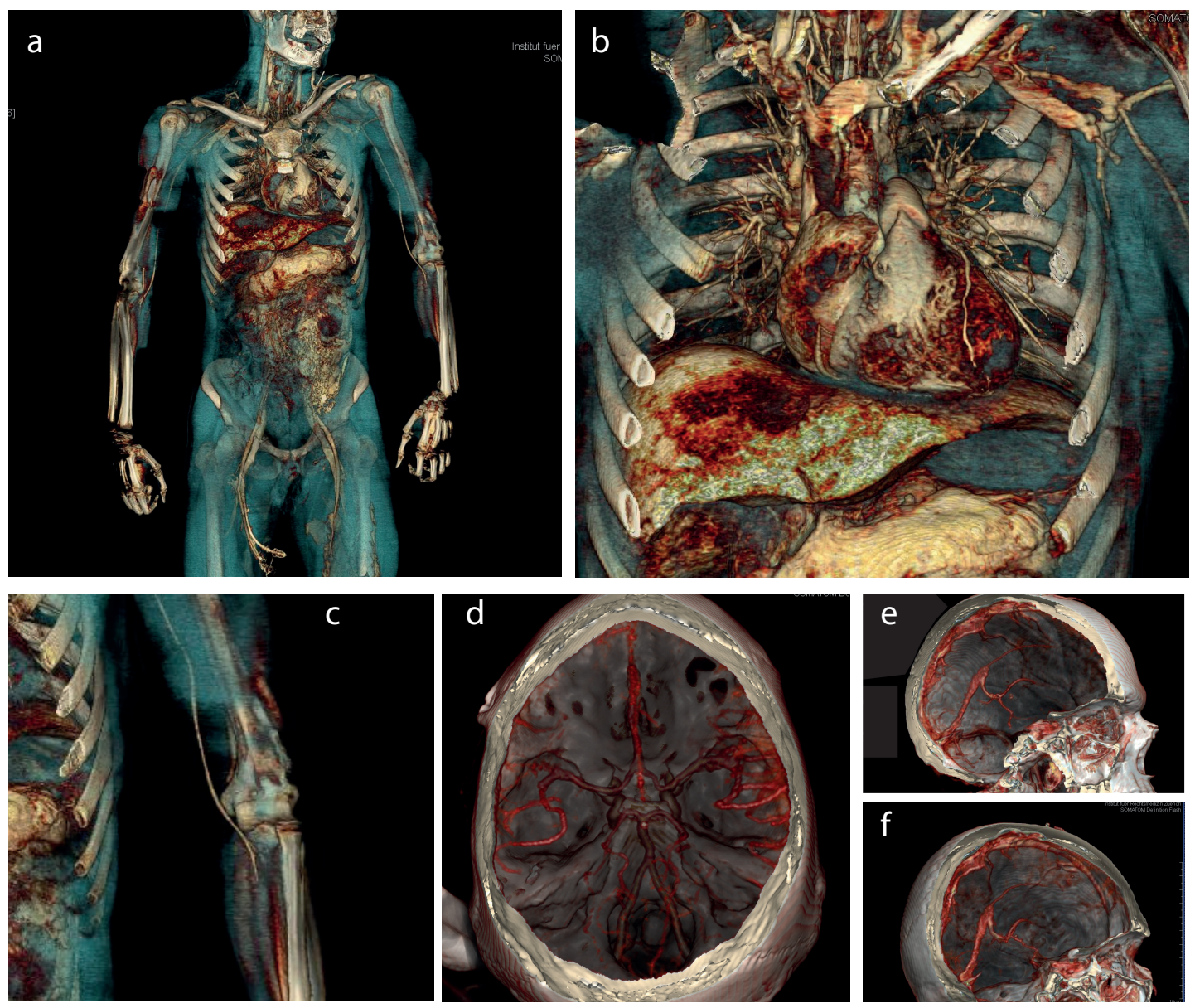

Figure 8: Resulting PMCTA with (a) view of whole body showing contrasted vessels and organs, (b) close-up of chest and upper abdomen with contrasted heart, coronary arteries, pulmonary veins and the liver, $(\boldsymbol{c})$ brachial artery filled past the elbow, $(\boldsymbol{d})$ intracranial arteries and $(\boldsymbol{e}, \boldsymbol{f})$ veins with excellent filling right to the end of the superior sagittal sinus. There is a large contrast leak into abdominal cavity on the left side due to hemorrhage. All images: VRT.

\begin{tabular}{l|lllll}
\hline Part & Supports $^{1}$ & Rafts $^{1}$ & infill[\%] & wt[g] & print[h] \\
\hline \hline Femoral catheter & yes & yes & $50 \%$ & 20.8 & $02: 55$ \\
\hline Compression fit & no & no & $10 \%$ & 3.1 & $00: 20$ \\
\hline VTS $^{2}$ stylet & yes & yes & $50 \%$ & 15.9 & $01: 33$ \\
\hline VTS $^{2}$ occl. tube & no & no & $10 \%$ & 11.9 & $01: 37$ \\
\hline VTS $^{2}$ quick clamp & yes & yes & $20 \%$ & 7.6 & $01: 12$ \\
\hline Tube fixture & yes & yes & $10 \%$ & 60.6 & $04: 00$
\end{tabular}

Table 1: 3D-print characteristics of parts (see Fig. 2) on a Makerbot Replicator+ $\left(5^{\text {th }}\right.$ gen), total $\sim 120$ g. $-{ }^{1}$ Supports or rafts necessary or not. ${ }^{2}$ Vascular tourniquet set. 

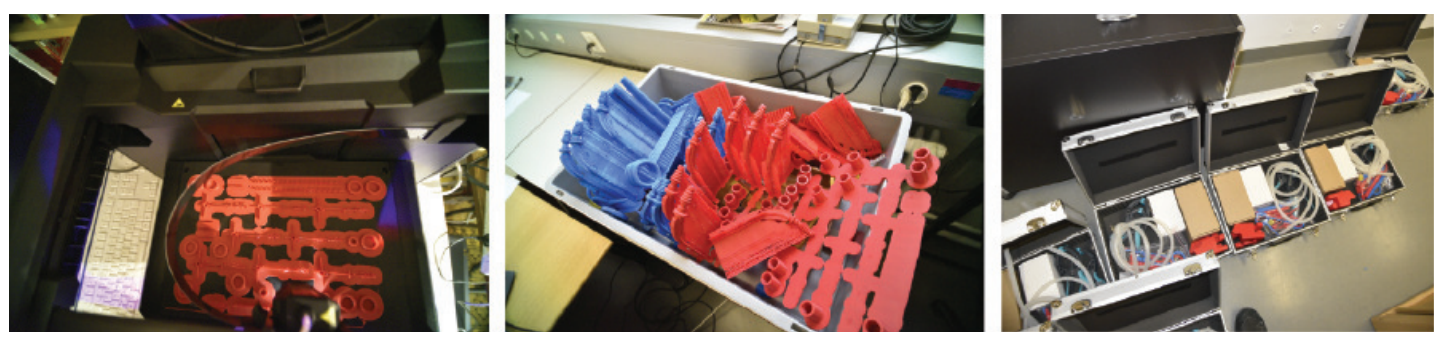

Figure 9: Production of Very Affordable PMCTA-kits - Left: 3D-printing in progress; Middle: finished print batches; Right: kits in process of being packed (preparation for Virtopsy Practical Course 2017).

\section{Discussion}

Once a product is validated and known to be affordable, and to function, previously negative individual-level beliefs can safely be updated to allow device adoption and usage [25].

In this instance, a very affordable PMCTA kit was created through the methods of rapid prototyping. With this, and in particular with the $3 \mathrm{~d}$-printed spring clamp tube fixture, significant handling problems of the isolated immersion pump that were initially pointed out (i.e., requiring bi-manual handling [12]) have been successfully remedied; there, PEG with contrast agent had spilled on the floor on occasion with one-handed operation.

All material specifications have been made freely available on-line (www.virtopsy.com). The industrially available materials can be purchased according to their specification as they are ubiquitous hardware store derived items. The 3D-parts that we had designed are freely downloadable as STL-file and 3d-printed, by anyone. Finished plastic parts can also be obtained from these 3D-models as 3d-printed items through any 3D print service website, without further overhead cost, in various higher-grade materials, such as acrylic, at the discretion of the user.

The kit that resulted from the requests of Virtopsy Course participants is very mobile: it fits into a small suitcase and is neither large or heavy. And yet, further device integration steps are possible as well. The whole kit may be used to build a sturdy frame around it, add large display options [26], make tube exchange faster and easier, and take the design integration even further ergonomically [27, 28].

\section{Conclusion}

It is possible to perform PMCTA with a superb vascular filling using very low cost equipment if one cares about the relevant technical details that are necessary to obtain good PMCTA contrast $[14,15]$. As a result, a parts specification of a PMCTA kit 
has been assembled by the authors that may be physically put together by anyone who wishes to do so at similarly low cost.

\section{References}

[1] Saiful Nizam bin Abdul Rashid, Heinrich Bouwer, and Chris O’Donnell. Lethal hemorrhage from a ureteric-arterial-enteric fistula diagnosed by postmortem ct angiography. Forensic Science, Medicine, and Pathology, 8(4):430-435, 2012.

[2] F-Z Mokrane, F Savall, C Rérolle, A Blanc, P Saint Martin, H Rousseau, D Rougé, $\mathrm{N}$ Telmon, and F Dedouit. The usefulness of post-mortem ct angiography in injuries caused by falling from considerable heights: three fatal cases. Diagnostic and Interventional Imaging, 95(11):1085-1090, 2014.

[3] Felipe Barjud Pereira do Nascimento, Glaucia Aparecida Bento dos Santos, Nelson Almeida dâĂŹAvila Melo, Eduarda Bittencourt Damasceno, and Thais Mauad. Detection of the source of hemorrhage using postmortem computerized tomographic angiography in a case of a giant juvenile nasopharyngeal angiofibroma after surgical treatment. Forensic Science, Medicine, and Pathology, 11(3):427-431, 2015.

[4] Frederic Savall, Fabrice Dedouit, Fatima-Zohra Mokrane, Daniel Rougé, Pauline Saint-Martin, and Norbert Telmon. An unusual homicidal stab wound of the cervical spinal cord: a single case examined by post-mortem computed tomography angiography (pmcta). Forensic Science International, 254:e18-e21, 2015.

[5] J Fornaro, P Laberke, and M Thali. Dual-phase post-mortem digital subtraction ct angiography: Color encoded demonstration of the arterial and venous phases. Journal of Forensic Radiology and Imaging, 3(2):134-135, 2015.

[6] L Sarda-Quarello, C Bartoli, PE Laurent, J Torrents, MD Piercecchi-Marti, S Sigaudy, D Ariey-Bonnet, and G Gorincour. Whole body perinatal postmortem ct angiography. Diagnostic \& Interventional Imaging, 97(1):121-4, 2016.

[7] Philipp Hinderberger, Barbara Fliss, Michael J Thali, and Wolf Schweitzer. Fatal bronchovascular fistula after lobectomy visualized through postmortem computed tomography angiography: A case report. Forensic Science, Medicine, and Pathology, 13(2):234-239, 2017.

[8] Axel Heinemann, Silke Grabherr, Fabrice Dedouit, Krzysztof Woźniak, Florian Fischer, Holger Wittig, Jochen Grimm, Hermann Vogel, et al. The impact of multiphase post-mortem ct-angiography (mpmcta) for investigating fatal outcomes of medical interventions. Journal of Forensic Radiology and Imaging, 8:38-44, 2017. 
[9] Mahmoud Hussami, Silke Grabherr, Reto A Meuli, and Sabine Schmidt. Severe pelvic injury: vascular lesions detected by ante-and post-mortem contrast medium-enhanced ct and associations with pelvic fractures. International Journal of Legal Medicine, 131(3):731-738, 2017.

[10] Damaris Fröhlich Knaute, Michael J Thali, Dominic Gascho, and Wolf Schweitzer. post mortem ct angiography of iliacofemoral arteries after cardiovascular surgery. Journal of Forensic Radiology and Imaging, 9:40-43, 2017.

[11] Sabine Franckenberg, Patricia M Flach, Dominic Gascho, Michael J Thali, and Steffen G Ross. Postmortem computed tomography-angiography (pmcta) in decomposed bodies-a feasibility study. Journal of Forensic Radiology and Imaging, 3(4):226-234, 2015.

[12] Wolf Schweitzer, Patricia Mildred Flach, Michael Thali, Patrick Laberke, and Dominic Gascho. Very economical immersion pump feasibility for postmortem CT angiography. Journal of Forensic Radiology and Imaging, 5:8-14, 2016.

[13] Steffen Ross, Danny Spendlove, Stephan Bolliger, Andreas Christe, Lars Oesterhelweg, Silke Grabherr, Michael J Thali, and Erich Gygax. Postmortem wholebody ct angiography: evaluation of two contrast media solutions. American Journal of Roentgenology, 190(5):1380-1389, 2008.

[14] Wolf Schweitzer, Patricia M Flach, Dominic Gascho, Lars Ebert, and Michael J Thali. Very Affordable Immersion Pump for Post Mortem CT Angiography in Forensic Pathology: First 10 Cases. In Forensic Science International, volume 277, page 22, 2017.

[15] Wolf Schweitzer, Dominic Gascho, Lars Ebert, Jakob Heimer, and Michael Thali. Virtopsy - economical centrifugal immersion pump outperforms heart-lung machine in 10 cases of post mortem ct angiography. In European Congress of Radiology, editor, ECR Online, Vienna, Austria; https : / / ecronline.myesr.org/ecr2018/index.php?p=recorddetail\& rid=48d94b73-3e0c-4740-823e-1213cbe1a539, 2018.

[16] S. Grabherr, J.M. Grimm, and A. Heinemann. Atlas of Postmortem Angiography. Springer International Publishing, Berlin, Germany, 2016.

[17] Shirley Taylor and Peter A Todd. Understanding information technology usage: A test of competing models. Information Systems Research, 6(2):144-176, 1995.

[18] Wolf Schweitzer, Michael Thali, Rosa Maria Martinez, and Lars Ebert. Post mortem ct angiography in fetuses or newborn: Very affordable pump and catheter solution. Journal of Forensic Radiology and Imaging, 13:17-22, 2018. 
[19] Sarah L Saunders, Bruno Morgan, Vimal Raj, and Guy N Rutty. Post-mortem computed tomography angiography: past, present and future. Forensic Science, Medicine, and Pathology, 7(3):271-277, 2011.

[20] Kaufui V Wong and Aldo Hernandez. A review of additive manufacturing. ISRN Mechanical Engineering, 2012, 2012.

[21] Wei Gao, Yunbo Zhang, Devarajan Ramanujan, Karthik Ramani, Yong Chen, Christopher B Williams, Charlie CL Wang, Yung C Shin, Song Zhang, and Pablo D Zavattieri. The status, challenges, and future of additive manufacturing in engineering. Computer-Aided Design, 69:65-89, 2015.

[22] Patricia M Flach, Dominic Gascho, Wolf Schweitzer, Thomas D Ruder, Nicole Berger, Steffen G Ross, Michael J Thali, and Garyfalia Ampanozi. Imaging in forensic radiology: an illustrated guide for postmortem computed tomography technique and protocols. Forensic Science, Medicine, and Pathology, 10(4):583606, 2014.

[23] Samantha Higgins, Sarah Parsons, Noel Woodford, Matthew Lynch, Christopher Briggs, and Chris O'Donnell. The effect of post-mortem computed tomography angiography (pmcta) using water-soluble, iodine-based radiographic contrast on histological analysis of the liver, kidneys and left ventricle of the heart. Forensic Science, Medicine and Pathology, 13(3):317-327, 2017.

[24] Benjamin Bax and Jörg Müssig. Impact and tensile properties of PLA/Cordenka and PLA/Flax composites. Composites Science and Technology, 68(7-8):16011607, 2008.

[25] Carlos A Trujillo, Andrés Barrios, Sonia M Camacho, and José Antonio Rosa. Low socioeconomic class and consumer complexity expectations for new product technology. Journal of Business Research, 63(6):538-547, 2010.

[26] Ravin Balakrishnan, George W Fitzmaurice, and Gordon Kurtenbach. User interfaces for volumetric displays. Computer, 34(3):37-45, 2001.

[27] Max Wintermark, Pierre-Alexandre Poletti, Christoph D Becker, and Pierre Schnyder. Traumatic injuries: organization and ergonomics of imaging in the emergency environment. European Radiology, 12(5):959-968, 2002.

[28] Jamaal L Benjamin and Quinn C Meisinger. Ergonomics in the development and prevention of musculoskeletal injury in interventional radiologists. Techniques in Vascular and Interventional Radiology, 21(1):16-20, 2018. 


\section{Acknowledgements}

The authors express their gratitude to Emma Louise Kessler, MD for her generous donation to the Zurich Institute of Forensic Medicine, University of Zurich, Switzerland. The first author expresses gratitude to Mr. Stephan Müller, Senior Manager, SHL Tooling TW, Taoyuan City, Taiwan for transfer of relevant build and CAD design knowhow. The authors also thank Dominic Gascho for supporting the PMCTA examination.

\section{Conflict of interest}

The authors have no commercial interest and no patents on the devices described; all designs have been made publicly accessible.

\section{Ethics approval}

Ethics approval of Cantonal Ethics Committee (Zuerich, Switzerland) was obtained (15-0686). 